\title{
Über die Verbindungen \\ der Metallrhodanide mit organischen Basen.
}

\author{
II. Mitteilung. \\ Von \\ Hermann Grossmann und Bernhard Schück.
}

Die Additionsverbindungen organischer Basen an anorganische Metallsalze zeigen zwar sehr verschiedene Zusammensetzung, ${ }^{1}$ lassen sich jedoch alle dem WerNerschen System einfügen. Wie in einer ersten Mitteilung ${ }^{2}$ gezeigt werden konnte, bestehen sicher Beziehungen zwischen den Molekulargewichten der organischen Basen und dem Typus der Additionsverbindungen. Andererseits ergab sich, dafs der Charakter der Base, ob stärker negativ oder nicht, eine Rolle spiele. Denn während stets nur $2 \mathrm{Mol}$. Anilin an Salze zweiwertiger Metalle addiert wurden, gelang bei den Rhodaniden speziell die Darstellung von Verbindungen mit $6 \mathrm{Mol}$. Phenylhydrazin, also einer Base mit gröfserem Molekulargewicht.

Ebenso beeinflufst der Säurerest wie das als Zentralatom wirkende Metall die Zusammensetzung der im festen Zustand bei gewöhnlicher Temperatur existenzfähigen Molekularverbindungen.

Um diese Beziehungen noch weiter zu prüfen, haben wir uns mit der Einwirkung einer zweiwertigen Base von niedrigem Molekulargewicht beschäftigt und speziell das Verhalten des Äthylendiamins gegen Rhodanide zweiwertiger Metalle studiert. Da Ảthylendiamin 2 Koordinationsstellen besetzt, so ist 3 die Maximalzahl der angelagerten Moleküle, wie bereits die Untersuchungen von WERNER ${ }^{3}$

' Reitzenstein, Z. anorg. Chem. 18 (1898), 296 und Renz, Z. anorg. Chem. 36 (1908), 110.

2 Z. anorg. Chem. 46 (1905), 361.

${ }^{3}$ Z. anorg. Chem. 21 (1899), 201.

z. anorg. Chem. Bd. 50. 
experimentell zeigten. Von Additionsverbindungen waren im wesentlichen Haloidsalze, Nitrate und Sulfate untersucht worden und nur eine Rhodanidverbindung, das Diäthylendiaminnickelrhodanid, das nach WERNER in 2 isomeren Modifikationen erhalten werden kann, war bisher bekannt. Wir konnten nun zeigen, dals die Rhodanidverbindungen im wesentlichen den Haloidsalzen entsprechen. Ferner ergab sich ganz allgemein die maximale Additionsfähigkeit beim Äthylendiamin als unabhängig von Metall- und Säurerest, da Nickel, Kupfer, Kobalt, Zink und Kadmiumsalze sehr leicht Verbindungen vom Grenztypus (Me3 en) $\mathrm{X}_{2}$ bilden, ja diese Grenzverbindungen sind beim Zink und Kadmium die einzig existenzfähigen. Wir konnten ferner zeigen, dals auch beim zweiwertigen Kupfer wie beim Nickel 3 Reihen von Salzen existieren, welche entsprechend den 3 WERNERschen Haupttypen, abgesehen vom Wassergehalt die Zusammensetzung $\mathrm{CuX}_{2} 1 \mathrm{en}, \mathrm{CuX}_{2} 2$ en und $\mathrm{CuX}_{2} 3$ en haben. Die Versuche über die Einwirkung der beiden hochmolekularen Basen $\alpha$ und $\beta$ Naphthylamin auf Rhodanide ergaben nur beim Nickel isolierbare Additionsverbindungen von der Formel $\mathrm{Ni}(\mathrm{SCN})_{2} 2 \alpha(\beta) \mathrm{C}_{10} \mathrm{H}_{7} \mathrm{NH}_{2}$.

\section{Experimenteller Teil.}

\section{Analytische Methoden.}

Bei den Analysen der dargestellten Körper geschah die Bestimmung der Metalle grölstenteils als Sulfate. Dazu wurde der Substanz zur Beschleunigung der Oxydation der organischen Bestandteile und zur Vermeidung zu grofser Verkohlung einige Tropfen konzentrierter $\mathrm{HNO}_{3}$ (spezifisches Gewicht 1.4) zugefügt, und dann die Substanz mehrmals mit konzentrierter Schwefelsäure vorsichtig abgeraucht, bis sie frei von organischen Resten war. Nach dieser Methode, die gute Resultate lieferte, wurden Mangan, Nickel, Kobalt, zum Teil auch Kadmium und Zink bestimmt, während sonst in gewöhnlicher Weise $\mathrm{Zn}, \mathrm{Cd}, \mathrm{Hg}$ und $\mathrm{Cu}$ als Sulfide zur Wägung gelangten. In allen Fällen konnte in den Additionsverbindungen die Stickstoffbestimmung sowie die organische Elementaranalyse vermieden werden, indem die Bestimmung des freien Äthylendiamins ${ }^{1}$

1 Die zu den Versuchen von de Ha ën bezogene $10 \%$ ige Lösung Äthylendiamin wurde titrimetrisch mit $1 / 10$ n. $\mathrm{H}_{2} \mathrm{SO}_{4}$ und Methylorange kontrolliert und erwies die Genaugkeit der Konzeutration:

$$
\left.\begin{array}{llll}
10 \mathrm{ccm} \text { en } 13.45 \mathrm{ccm} 1 / 10 & \text { n. } \mathrm{H}_{2} \mathrm{SO}_{4}=9.977 \% \text { en } \\
10 \mathrm{ccm} \text { en } 13.40 \mathrm{ccm} 1 / 10 & \text { n. } \mathrm{H}_{2} \mathrm{SO}_{4}=9.978 \% \text { en }
\end{array}\right\} \text { statt } 10 \% \text {. }
$$


und des $\ddot{A}$ thylendiamins in den Additionsverbindungen azidimetrisch ansgeführt wurde. Dieser Befund steht im Gegensatz zu der Ansicht von Ostwali, ${ }^{1}$ dem die Titration nicht gelang. Jedoch bei der stärkeren basischen Natur des Äthylendiamins gegenüber dem Ammoniak ist die Ausführbarkeit dieser Bestimmungsmethode leicht erklärlich und lieferte bei Benutzung von $1 / 10-n$ Schwefelsäure zur Neutralisation und Methylorange oder Lackmus als Indikator genaue Resultate, während mit Phenolphtalein als Indikator der Übergang unscharf and nur die Hälfte $1 / 10^{-n} \mathrm{H}_{2} \mathrm{SO}_{4}$ zur Neutralisation erforderlich war.

Die zweite Hydroxylgruppe in dem $\ddot{A}$ thylendiammoniumbydroxyd $\mathrm{CH}_{2} \mathrm{NH}_{3} \mathrm{OH}$ $\mathrm{CH}_{2}, \mathrm{NH}_{3} \mathrm{OH}$, das wir in der wässerigen Lösung des Äthylendiamins anzunehmen haben, ist demnach erheblich schwächer basisch und verhält sich etwa wie das 2. und 3. Wasserstoffatom in der Phosphorsäure: Der Vorgang bei der Titration des Ätbylendiamins in den Additionsverbindungen lärst sich wohl so erklären, dafs auch das komplex gebundene Äthylendiamin, welches hier wie die freie Base reagiert, einfach neutralisiert wird, so dafs am Neutralisationspunkt sich das Metallsalz und eine neutrale Äthylendiammoniumverbindung in Lösung befindet, z. B.:

$$
\left.(\mathrm{Ni} \mathrm{en})_{3}\right) \mathrm{Cl}_{2}+6 \mathrm{HCl}=3\left(\mathrm{H}_{2} \text { en }\right) \mathrm{Cl}_{2}+\mathrm{NiCl}_{2} .
$$

Die titrimetrische Bestimmung des Äthylendiamins war sogar bei gefärbten Salzen anwendbar; bei den blaugefärbten Kupfersalzen z. B. brachte der Zusatz von $\mathrm{H}_{2} \mathrm{SO}_{4}$ zuerst Farbenumschlag in grün hervor; in der Nähe des Neutralisationspunktes, wo nur geringe Mengen der Komplexverbindungen vorhanden waren, wurde die Lösung gelb, so dafs in genügender Verdünnung der Farbenumschlag scharf erkennbar war, da die schwache hellblaue Färbung der KupriIonen nicht störte.

Leider ist dieses bequeme Titrationsverfahren nur auf die Additionsverbindungen von Nickel, Kupfer, Zink und Kadmiumsalzen beschränkt. Weder das an Kobalt und Platin, noch das an Säure gebundene Ätbylendiamin der Äthylendiammoniumsalze läfst sich wie Ammoniak durch Kochen mit Kalilauge überdestillieren und titrimetrisch bestimmen, da der Siedepunkt zu hoch liegt (116.5 ) und die Destillation bei hoher Temperatur Zersetzung hervorruft.

1 Journ. prakt. Chem. [2] 33 (1856), 268. 


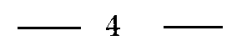

\section{Nickelverbindungen. ${ }^{1}$}

a) Rhodanide.

Triäthylendiamin-Nickelrhodanid.

$$
\mathrm{Ni}(\mathrm{SCN})_{2} \cdot 3\left(\begin{array}{l}
\mathrm{CH}_{2} \mathrm{NH}_{2} \\
\mathrm{CH}_{2} \mathrm{NH}_{2}
\end{array}\right)
$$

Setzt man zu einer konzentrierten Lösung ron Nickelrhodanid langsam, unter stetigem Umrühren die dreifach molekulare Menge Äthylendiamin, so entsteht zunächst ein blaugrüner Niederschlag, der sich bald im Überschufs des Äthylendiamins mit blauvioletter Farbe löst; und aus der schliefslich rotviolett gefärbten Lösung kristallisiert das Salz nach genügender Konzentration in violetten, prismatischen Kristallen, die von der Mutterlauge getrennt und getrocknet wurden.

Schmelzpunkt $253^{\circ}$.

Das Salz ist ziemlich beständig und in heifsem Wasser leicht, in kaltem märsig leicht mit rotvioletter Farbe löslich. Säuren zerstören seine Komplexität, indem das Äthylendiamin in Form des Äthylendiammoniumsalzes der Säure abgespalten wird. Da das Äthylendiammonium-Ion farblos ist, so erscheint dann die Lösung nur durch die Nickelionen gefärbt. Während $\mathrm{KOH}$ nur in der Hitze grünes Nickelhydroxyd fällt, ist beim Ammoniak auch in der Hitze keine Fällung bemerkbar, da das Hexamminsalz eine sehr beständige Komplexverbindung ist, die nur wenig Nickelionen entbält. Schwefelwasserstoff fällt sehwarzes Sulfid, dagegen rufen Natriumkarbonat und Natriumphosphat $\left(\mathrm{Na}_{2} \mathrm{HPO}_{4}\right)$ keinen Niederschlag hervor, Ferrocyankalium gibt eine schwache Trübung, während Ferricyankalium die Lösung braun färbt, aus der nach einiger Zeit ein brauner Niederschlag fällt. Cyankalium färbt die konzentrierte Lösung orangegelb; aus ihr kristallisiert beim Abkühlen das später behandelte Cyanid aus.

1 Die Bearbeitung dieser Verbindungen ist in der Reihenfolge ihrer Atomgewichte angefüht, und zwar wurden zuerst die Additionsverbindungen der Rhodanide mit Äthylendiamin beschrieben, während im Anschlusse daran noch einige Additionsprodukte mit anderen Metallsalzen behandelt wurden, da es zweckmälsig erschien, auch diese Reihen durch Darstellung der noch fehlenden Verbindungen zu ergänzen. 


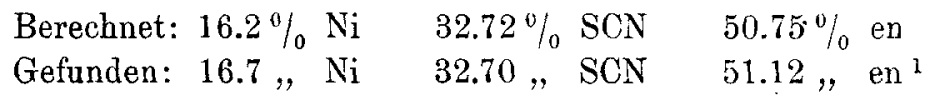

Diäthylendiamin-Nickelrhodanid.

Dieses Salz gewinnt an Interesse durch den Isomeriefall, den WERNER ${ }^{2}$ bei seiner Darstellung beobachtet haben will, und zu dessen Klärung die mehrfache Darstellung und Untersuchung dieser Verbindung ausgeführt wurde. WERNER ging aus vom Diäthylendiamin-Nickelbromid, das er mit festem Kaliumrhodanid umsetzte; den entstehenden violettroten Niederschlag kristallisierte er aus Wasser um und erbielt zunächst schwerer lösliche blauviolette, sechsseitige Platten, während sich als weiteres Kristallisationsprodukt rosaviolette Nadeln abschieden. Beide wurden einzeln nochmals aus Wasser umkristallisiert und ergaben übereinstimmende Analysen. $\left[\mathrm{Ni}(\mathrm{SCN})_{2} 2 \mathrm{en}\right]+1 \mathrm{aq}$. Er betrachtete diese Körper als isomer.

Zur Lösung dieser interessanten, aber wenig geklärten Frage unternahmen wir die Darstellung des Körpers auf zwei Wegen. Durch direkte Einwirkung des Äthylendiamins auf Nickelrhodanid und durch Umsetzung mittels KCNS aus den entsprechenden Nickelsulfat- und Nickelchloridsalzen.

\section{Direkter Weg.}

Die grüne Farbe der konzentrierten Nickelrhodanidlösung nahm auf Zusatz der molekularen Menge Äthylendiamin eine blauviolette Farbe an, und aus ihr kristallisierten nach Konzentration teils nebeneinander, teils nacheinander beim Abkühlen blaue und rosaviolette Kristalle. Sie wurden mechanisch getrennt und zeigten beide den Schmelzpunkt $220^{\circ}$. Nur die blauen Kristalle waren der kristallographischen Messung zugänglich, die Herr A. H. WesterGöRD auf Veranlassung von Herrn Dr. H. Steinmetz im Münchener

1 Bei der Angabe der gefundenen Werte ist stets das Mittel aus den Einzelbestimmungen angegeben worden. Die Metallbestimmung geschah als Sulfat in der vorher angegebenen Weise durch Abrauchen mit $\mathrm{H}_{2} \mathrm{SO}_{4}$; die Äthylendiamin-Analyse titrimetrisch mit $\mathrm{H}_{2} \mathrm{SO}_{4}$ (Titer 0.989 1/10 n.) und Methylorange als Indikator in allen Additionsverbindungen. Die Rhodanbestimmung wurde ebenfalls titrimetrisch ausgeführt, indem man die Lösung der Substanz mit Ferrisalz versetzte und $1 / 10$ n. AgNO, zufliefsen liefs. In allen Fällen, wo dies nicht möglich war, geschah sie durch Oxydation des Schwefels zu Schwefelsä.ure und Bestimmung desselben als $\mathrm{BaSO}_{4}$. Vergl, auch $\mathrm{B}$. Scнück, Inauguraldissertation, Münster 1906.

\& Z. anorg. Chem. 21 (1899), 231. 
mineralogischen Institut von Prof. voN GronH ausführte, wofür wir unseren besten Dank auch an dieser Stelle aussprechen wollen.

Monoklin: $a: b: c==1.2371: 1: 1.8304$.

$$
\beta=94^{\circ} 14^{\prime} \text {. }
$$

Beobachtete Formen: a $\{100\}, o\{001\}, r^{\prime}\{101\}, g\{301\}, o\{111\}$, $o^{\prime}\{111\}, x\{121\}, y\{\overline{2} 25\}$, und einige andere positive Hemipyramiden mit komplizierten Indices.

Dünntafelig nach der Basis. Als zeitliche Begrenzung treten die Flächen (12) wodurch die Kristalle einen pseudotrigonalen Habitus erhalten. Die Pyramiden mit komplizierteren Indices sind klein und matt.

\begin{tabular}{|c|c|}
\hline Beobachtet: & Berechnet: \\
\hline$(100):(001)={ }^{*} 85^{0} 46^{\prime}$ & - \\
\hline$(001):(\overline{1} 01)=59^{\circ} 02^{\prime}$ & $58^{0} 53^{\prime}$ \\
\hline$(001):(301)=73^{0} 45^{\prime}$ & $73^{0} 18^{\prime}$ \\
\hline$(001):(111)=64^{0} 51^{\prime}$ & $64^{0} 42^{\prime}$ \\
\hline$(001):(\overline{1} 11)=69^{\circ} 20^{\prime}$ & $69^{\circ} 13^{\prime}$ \\
\hline$(001):(121)=* 74^{\circ} 16^{\prime}$ & - \\
\hline$(121):(: \overline{2}])=126^{\circ} 27^{\circ}$ & $126^{\circ} 21^{\prime}$ \\
\hline$(121):(100)={ }^{*} 67^{0} 41^{\prime}$ & - \\
\hline$(111):(\overline{1} 11)=71^{\circ} 12^{\prime}$ & $70^{0} 48^{\prime}$ \\
\hline$(001):(\overline{2} 25)=66^{\circ} 40^{\prime}$ & $67^{\circ} 16^{\prime}$ \\
\hline
\end{tabular}

Vollkommene Spaltbarkeit nach $\{001\}$.

Ebene der optischen Achsen ist $\{010\}$. Auf $e$ nahe an $\{100\}$ eine Achse sichtbar.

Deutlich pleochroitisch; auf $e$ sind die Schwingungen parallel der Symmetrieebene hell lavendelblau, senkrecht dazu violett.

Einige Reaktionen der blauen Verbindung, die mit denen der roten vollkommen identisch waren, seien hier angeführt. Beide Salze waren in Wasser leicht löslich und wurden durch $\mathrm{KOH}$, nicht durch Ammoniak gefällt. Auch Natriumphosphat war unwirksam, während Quecksilberchlorid einen in der Hitze löslichen, weifsen Niederschlag, Ferrocyankalium einen hellgrünen Niederschlag, und Ferricyankalium in der Hitze einen braunen Niederschlag herrorriefen.

Berechnet: $19.8 \% \mathrm{Ni}$

Gefunden: $19.45 \% \mathrm{Ni}$
$40.72 \%$ en $40.42 \%$ en
$39.35 \%$ SCN

$39.32 \%$ SCN 
Im Gegensatz zu der Wernenschen Verbindung $\left(\mathrm{Ni} \mathrm{en}_{2}\right)(\mathrm{SCN})_{2}$ + 1 aq war also der Körper wasserfrei. Dasselbe Resultat ergab auch die Darstellung durch direkte Einwirkung des Äthylendiamins auf festes Nickelrhodanid.

Nickelbestimmung $=19.51 \%$ und $\ddot{\text { Äthylendiamintitration }=}$ $40.43 \%$ identifizierten das Salz. Hier hingegen kristallisierten nur hellblau gefärbte Kristalle, und zu gleichem Resultate gelangt man auch durch Darstellung der Verbindung

\section{2. durch Umsetzung}

aus Diäthylendiamin-Nickelsulfat mittels einer molekularen Menge KCNS; denn die blau und rosa-violett gefärbten Kristalle, die jetzt kristallisierten, ergaben bei der Umkristallisation aus Wasser von $85^{\circ}$ beim Erkalten nur blaue Kristalle mit dem gleichen Schmelzpunkt $220^{\circ}$.

Auch hier bewies die $\ddot{A}$ thylendiaminbestimmung $=40.35 \%$, dals das Salz wasserfrei war.

Die Isomerie kann auf ähnliche Weise wie bei der Verbindung $\mathrm{Co}(\mathrm{SCN})_{2} \cdot 4 \mathrm{Py}$ von $\mathrm{S}_{\mathrm{AND}}{ }^{1}$ erklärt werden. In Betracht kämen ferner noch die beiden isomeren Formeln I $\left(\begin{array}{ll}\mathrm{Ni}_{\mathrm{SCN}} & \underset{\mathrm{Sn}}{2}\end{array}\right)$ und II $\left(\mathrm{Ni} \mathrm{en},(\mathrm{SCN})_{2}\right.$. Formel I leitet sich von der Oktaederformel ab und besitzt keine ionisierbaren Reste, II dagegen von der planen Formel, die für 2 wertige Metalle im allgemeinen die Koordinationszahl 4 als Grenzzahl annimmt und 2 ionisierbare Reste besitzt. ${ }^{2}$ Eine Entscheidung lärst sich leider bisher experimentell nicht treffen.

\section{Monoäthylendiamin-Nickelrhodanid. $\mathrm{Ni}(\mathrm{SCN})_{2} .1 \mathrm{en}$.}

Man erhält diese Verbindung am einfachsten durch Zusammenbringen molekularer Mengen von Äthylendiamin und Nickelrhodanid, wodurch die Lösung eine stahlbliue Farbe annimmt. Aus ihr kristallisiert das Salz nach 24 stündigem Stehen im Vakuumexsikkator in blaugrünen rhombischen Kristallen.

Schmelzpunkt $275^{\circ}$.

Schwer in kaltem, leicht in warmem Wasser löslich, $\mathrm{KOH}$ fällt erst in der Hitze hellgrünes Hydroxyd, Schwefelwasserstoff schwarzes Sulfid, dagegen ergaben Ammoniak und Natriumphosphat keinen

1 Ber. deutseh. chem. Ges. 36 (1903), 1436.

2 Werner, Z, anorg. Chem. 3 (1898). 
Niederschlag, während Ferrocyankalium einen im Überschusse löslichen, weirsen Niederschlag, Ferricyankalium schon in der Kälte eine braune Fällung hervorriefen. Quecksilberchlorid verursachte einen weifsen Niederschlag.

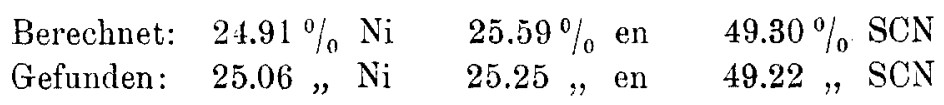

b) Cyanide und Haloide.

Triäthylendiamin-Nickelcyanid. $\mathrm{Ni}(\mathrm{CN})_{2} .3 \mathrm{en}$.

Als Analogon des Triäthylendiamin-Nickelrhodanids wurde diese Verbindung durch Zufügen der molekularen Menge von festem KCN zu Diäthylendiamin-Nickelrhodanid dargestellt. Aus der konzentrierten Lösung kristallisierte schon nach kurzer Zeit das rosaviolett gefärbte Salz in prismatischer Form aus.

Schmelzpunkt: $244^{\circ}$.

Das Salz unterscheidet sich von dem Rhodanid durch seine geringere Löslichkeit in Wasser und Alkohol, sowie durch eine gelbe Fällung mit Ferricyankalium. Bromwasser bewirkt Entfärbung der Lösung und Quecksilberchlorid einen weifsen, in der Hitze löslichen Niederschlag. Es ist bemerkenswert für die Stabilität des Komplexes $\left(\mathrm{Ni} \mathrm{en}_{3}\right) \mathrm{R}_{2}$, dals bei der Einwirkung von $\mathrm{KCN}$ sich nicht das komplexe $\mathrm{K}_{2} \mathrm{NiCN}_{4}$ bildet, sondern einfach Umsetzung zwischen SCN und CN stattfindet. $\begin{array}{ll}\text { Berechnet: } 20.17 \% \mathrm{Ni} & 61.92 \% \text { en } \\ \text { Gefunden: } 20.13 \% \mathrm{Ni} & 61.35 \% \text { en }\end{array}$

Diäthylendiamin-Nickelchlorid. $\mathrm{NiCl}_{2} \cdot 2 \mathrm{en}+1 \mathrm{H}_{2} \mathrm{O}$.

Mit den Additionsverbindungen der Haloidsalze haben sich WERNER und seine Schüler beschäftigt und Verbindungen der Jodide und Bromide dargestellt. Bei keinem Haloid gelang bisher die Darstellung der Monoäthylendiaminverbindungen. Von den Chloriden untersuchten Werner und Sprdck nur das Trï̈thylendiamin-Nickelchlorid, welches wir gleichfalls erhalten habe. Seine Analyse ergab die Identität mit dem Salze von Werner und Sprdok. 
$\begin{array}{ll}\text { Berechnet: } 16.97 \% \mathrm{Ni} & 52.10 \% \text { en } \\ \text { Gefunden: } 17.09 \% \mathrm{Ni} & \mathbf{5 2 . 7 2 \%} \text { en }\end{array}$

Zur Herstellung der Diäthylendiaminverbindung wurde Nickel. chlorid in Wasser gelöst und 2 Mol. Äthylendiamin zugegeben, wobei Farbenwechsel der Lösung von grün nach violett eintrat. Diese violette Lösung wurde bis zur Kristallhaut eingedampft, worauf beim Erkalten das Tetraminsalz in hellblauen, in Wasser leicht löslichen, prismatischen Kristallen kristallisierte.

Schmelzpunkt: $157^{\circ}$.

Berechnet: $21.92 \% \mathrm{Ni}$ $26.50 \% \mathrm{Cl} \quad 44.81 \%$ en

Gefunden: $22.03, \mathrm{Ni}$ $26.31, \mathrm{Cl}$ 44.27, en

Die Versuche, eine Monoäthylendiamin-Nickelchloridverbindung darzustellen, verliefen resultatlos; es schied sich stets das obige Salz ab.

Man kennt demnach jetzt folgende Äthylendiaminadditions. produkte von Nickelsalzen.

I. Hexamminsalze:

$\left.(\mathrm{Ni} \mathrm{en})_{3}\right) \mathrm{Cl}_{2}+2 \mathrm{aq},\left(\mathrm{Ni} \mathrm{en}_{3}\right) \mathrm{Br}_{2}+2 \mathrm{aq},\left(\mathrm{Ni} \mathrm{en}_{3}\right) \mathrm{J}_{2}+\mathrm{aq},\left(\mathrm{Ni} \mathrm{en}_{3}\right)\left(\mathrm{SCN}_{2}\right.$, $(\mathrm{Ni} \mathrm{en},)(\mathrm{CN})_{2},\left(\mathrm{Ni} \mathrm{en}_{3}\right)\left(\mathrm{NO}_{3}\right)_{2},(\mathrm{Ni} \mathrm{en}) \mathrm{SO}_{4}$.

II. Tetramminsalze.

$\left(\begin{array}{c}\mathrm{Ni} \mathrm{H}_{2} \mathrm{O} \\ \mathrm{Cl}\end{array}\right) \mathrm{Cl},\left(\begin{array}{l}\mathrm{Ni} \\ \underset{\left(\mathrm{H}_{2} \mathrm{O}\right)_{2}}{\mathrm{en}_{2}}\end{array}\right) \mathrm{Br}_{2},\left(\begin{array}{l}\mathrm{Ni} \\ \underset{\left(\mathrm{H}_{2} \mathrm{O}\right)_{2}}{\mathrm{en}_{2}}\end{array}\right) J_{2}, \quad\left(\begin{array}{l}\mathrm{Ni} \\ \left.\mathrm{SCN}_{2}\right)_{2}\end{array}\right)(2$ Isomere $)$.

III. Diamminsalze:

$$
\left(\mathrm{Ni} \underset{(\mathrm{SCN})_{2}}{\mathrm{en}}\right), \quad\left(\mathrm{Ni}_{\left(\mathrm{H}_{2} \mathrm{O}\right)_{4}}^{\text {en }}\right) \mathrm{SO}_{4}+1 \text { aq. }
$$

Die Farbe der Hexamminsalze ist im allgemeinen dunkelviolett bis rot, die der Tetramminsalze hellviolett bis blau und die der Diamminsalze blau bis grün.

\section{Zink- und Kadmiumsalze.}

Charakteristisch für die Additionsverbindungen der Zink- und Kadmiumsalze, von denen Werner und seine Schüler die Chloride, Bromide und Jodide darstellten, ist die Tatsache, dals Di- und Tetramminverbindungen nicht existieren, da durch Zusatz von 
Äthylendiammin zuerst immer die entsprechenden Hydroxyde aus. fallen, die erst bei Zusatz von $3 \mathrm{Mol}$. Äthylendiammin in Lösung gehen. Der gleiche $\nabla$ organg findet bei der Darstellung der Additionsverbindungen der Zink- und Kadmiumrhodanide statt; auch hier wird zuerst das Hydroxyd gefällt und dieses löst sich dann durch weiteren Zusatz der Base.

Der Verlauf dieses Vorganges ist vielleicht analog der Reaktion ron Ammoniak auf Kupfersulfatlösung. Die bei der Darstellung des Triäthylendiamin-Zinkrhodanids verlaufenden Reaktionen wären daher folgende:

1. $\mathrm{Zn}(\mathrm{SCN})_{2}+\underset{\mathrm{CH}_{2}}{\mathrm{CH}_{2}} \cdot \mathrm{NH}_{2}+2 \mathrm{H}_{2} \mathrm{O}=\mathrm{Zn}\left(\mathrm{OH}_{2}\right)+\underset{\mathrm{CH}_{2}}{\mathrm{CH}_{2}} \cdot \mathrm{NH}_{3} \cdot \mathrm{SCN}$

$$
\text { 2. } \mathrm{Zn}(\mathrm{OH})_{2}+{ }_{\mathrm{CH}_{2}}^{2 \mathrm{CH}_{2}} \cdot \mathrm{NH}_{2}=\left[\mathrm{Z1}\left(\mathrm{CH}_{2} \cdot \mathrm{CH}_{2} \cdot \mathrm{NH}_{2}\right)_{2}\right](\mathrm{OH})_{2} \text {. }
$$

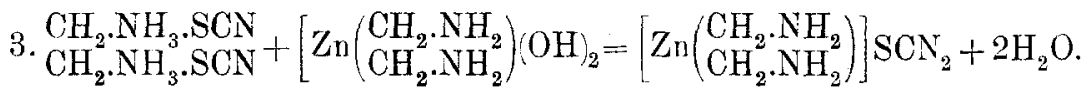

Das Äthylendiam in wirkt primär auf das Zinkrhodanid ein und es entsteht Zinkhydroxyd; letzteres ist aber eine viel zu schwache Base, um auf das đ̈thylendiammonium-Rhodanid einwirken zu können. Es mufs sich zuerst mit weiterem Äthylendiamin zu einer neuen,

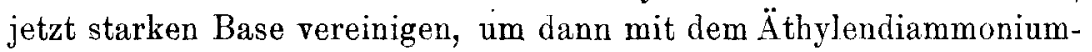
rhodanid nach Gleichung 3 reagieren zu können.

\section{Triäthylendiamin-Zinkrhodanid. $\mathrm{Zn}(\mathrm{SCN})_{2} .3 \mathrm{en}$.}

Die Darstellung des Salzes ist im obigen Abschnitt schon erläutert; die nach Zufügen von $3 \mathrm{Mol}$. Äthylendiamin zu Zinkrhodanid entstandene klare Lösung wird eingeengt, und aus ihr kristallisiert das Salz in weilsen, rhomboedrischen Kristallen.

Schmelzpunkt: $161^{\circ}$.

Diese Verbindung ist in Wasser sehr leicht löslich; aus der Lösung fällt $\mathrm{KOH}$ in der Hitze das weifse Hydroxyd, Schwefelwasserstoff weifses Zinksulfid, Ferrocyankalium einen unlöslichen Niederschlag, Ferricyankalium dagegen nichts. Auch durch Natriumphosphat entsteht in der Hitze ein weifser Niederschlag.

Berechnet: $18.07 \% \mathrm{Zn}$

Gefunden: $17.84, \mathrm{Zn}$
$49.81 \%$ en

50.16 , en
$32.11 \%$ SCN

31.94 .. SCN 
Triäthylendiamin-Kadmium rhodanid.

$\mathrm{Cd}(\mathrm{SCN})_{2} \cdot 3$ en.

Die Darstellung dieser Additionsverbindung geschah analog der des Zinksalzes. Wiederum fiel zuerst weilses Hydroxyd aus, das sich im Überschusse des Äthylendiamins löste. Aus der klaren Lösung kristallisierten nach längerem Stehen über Schwefelsäure weifse Platten.

Schmelzpunkt: $138^{\circ}$.

Seine Löslichkeit ist geringer als die des Zinksalzes, doch zeigt es im allgemeinen die gleichen Reaktionen wie jenes. Schwefelwasserstoff fällt gelbes Sulfid, Ammoniumkarbonat und Quecksilberchlorid in der Hitze weifse Niederschläge.

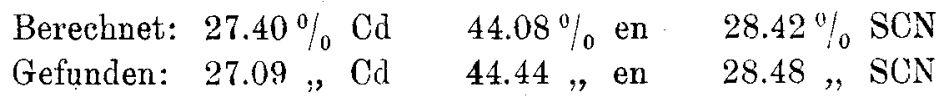

Entsprechend seiner starken basischen Natur vereinigt sich das Äthylendiamin nicht mit Mangan, Ferro- und Kobaltsalzen, wie das Pyridin und Chinolin. Es fällt wie Ammoniak aus Mangan- und Eisensalzen deren Hydroxyde, während Kobaltsalze zwar Komplexe bilden, die sich aber vom dreiwertigen Kobalt herleiten, äbnlich wie die Kobaltiake.

\section{Kupferverbindungen.}

Ebenso wie bei den Nickelsalzen finden wir auch beim Kupfer drei Reihen: Tri-, Di- und Mono-Äthylendiaminverbindungen, die sich voneinander in charakteristischer Weise chemisch und physikalisch unterscheiden. Die Tabelle am Schlufs des Abschnittes gibt eine Übersicht über die bisher dargestellten Additionsverbindungen, aus der ersichtlich ist, dals die Hexamminsalze am unbeständigsten sind; allerdings mufs hervorgehoben werden, dafs die Versuche rein qualitativer Art waren. Die Monoverbindungen, auf deren Vorhandensein die entsprechenden Nickelsalze schliefsen lassen, waren bisher nicht untersucht worden.

Die Reihen der Rhodanide, Bromide und Acetate stellten wir vollständig dar, während die Chloride, Sulfate und Nitrate nach dem vorliegenden Material ergänzt werden.

a) Rhodanide.

Die Darstellung der Additionsverbindungen der Kupferrhodanide mit $\ddot{A}$ thylendiamin geschah durch Umsetzung aus den entsprechen- 
den Bromiden, was leicht bei den Tri- und Diäthylendiaminsalzen gelang, bei der Monoverbindung aber an ihrer leichten Zersetzlichkeit in der Hitze scheiterte. Seine Darstellung geschieht besser in der Kälte aus dem entsprechenden Sulfat.

Triäthylendiamin-Kupferrhodanid.

$$
\mathrm{Cu}(\mathrm{SCN})_{2} \cdot 3 \text { en }+5 \mathrm{H}_{2} \mathrm{O} \text {. }
$$

Eine konzentrierte Lösung des Triäthylendiamin-Kupribromids (5 aq) wurde mit 2 Mol. KCNS versetzt und die blaue Lösung eingeengt. Das nach kurzer Zeit in tiefblauen, grofsen Platten kristallisierte Salz konnte leicht von dem weifsen $\mathrm{KBr}$ mechanisch getrennt und nochmals aus wenig Wasser umkristallisiert werden.

Schmelzpunkt: $138^{\circ}$.

Das Salz zeigt Beständigkeit, ist in Wasser leicht löslich, und weder auf Zusatz von $\mathrm{KOH}$ noch von $\mathrm{NH}_{3}$ tritt Hydroxydfällung ein, $\mathrm{H}_{2} \mathrm{~S}$ fällt wie gewöhnlich schwarzes Sulfid. Während Natriumphosphat, Natriumkarbonat und Jodkalium keinen Niederschlag geben, fällt Jod in Jodkalium einen in der Hitze unlöslichen, schwarzen, Ferrocyankalium in der Hitze einen rotbraunen, Quecksilberchlorid einen weilsen Niederschlag, der sich ebenfalls in der Hitze nicht löst. Bromwasser entfärbt wie KCN die Lösung und verursacht nach einiger Zeit eine grüne Trübung. Oxalsäure bringt Farbenumschlag in Hellblau hervor und fällt nach einiger Zeit einen Niederschlag von Kuprioxalat.

$\begin{array}{llll}\text { Berechnet: } & 14.13 \% \mathrm{Cu} & 14.20 \% \mathrm{~S} & 40.01 \% \\ \text { Gefunden: } & 13.84, \mathrm{Cu} & 13.88 \% \mathrm{~S} & 40.10 \% \text { en }\end{array}$

Diäthylendiamin-Kupferrhodanid.

$$
\mathrm{Cu}(\mathrm{SCN})_{2} \cdot 2 \mathrm{en}+2 \mathrm{H}_{2} \mathrm{O} \text {. }
$$

Nach Zusatz von $2 \mathrm{Mol}$. KCNS zu dem entsprechenden Diäthylendiamin-Kupferbromid (2aq) in alkoholischer Lösung kristallisierte nach Eindampfen bis zur Kristallhaut das Salz in dunkelblauen Nadeln neben farblosen $\mathrm{KBr}$ aus und wurde von letzterem durch Übergiefsen mit heifsem Alkohol getrennt. Hierdurch ging das Rhodanid in Lösung und schied sich aus ihr nach einiger Konzentration rein $a b$.

Schmelzpunkt: ca. $83^{\circ}$.

Es ist in Wasser leicht mit tief-violetter Farbe löslich und wird auch von Kalilauge und Ammoniak nicht beeinflufst. Jod in Jodkaliumlösung zeigt einen in der Hitze löslichen, dunkelgrünen, 
Silbernitrat einen hellrosa Niederschlag, der beim Erhitzen schmutzigbraun wird. $\mathrm{FeCl}_{3}$ gibt sofort die Rhodanreaktion; während Ferrocyankalium nur in der Hitze eine grüne Trübung hervorbringt, zeigt Ferricyankalium Farbenumschlag in grün. Cyankalium reduziert das Kupri- zum Kuprosalz unter Entfärbung und Cyanentwickelung.

Die durch $\mathrm{HgCl}_{2}$ hervorgebrachte gelbe Fällung wird beim Erhitzen kristallinisch.

$\begin{array}{llll}\text { Berechnet: } 18.93 \% \mathrm{Cu} & 19.01 \% \mathrm{~S} & 35.76 \% & \text { en } \\ \text { Gefunden: } & 18.84, \mathrm{Cu} & 19.31 \% \mathrm{~S} & 35.81 \% \text { en }\end{array}$

Monoäthylendiamin-Kupferrhodanid.

$$
\mathrm{Cu}(\mathrm{SCN})_{2} .1 \mathrm{en} \text {. }
$$

Ausgehend von dem Monoäthylendiamin-Kupfersulfat (3 aq) gelang die Darstellung dieses Körpers durch Umsetzung mit 2 Mol. Rhodankalium in der Kälte, während in der Wärme Zersetzung eintrat und sich teilweise unlösliches Kuprorhodanid abschied, woraus hervorgeht, dafs die Lösungen der Monosalze nicht unbeträchtliche Mengen von freien Kupriionen enthalten. Aus der blauen Lösung schied sich das Salz sofort in feinen, hellblauen Nadeln aus, die abgesaugt und aus viel Wasser nochmals umkristallisiert wurden. Die Umsetzung aus dem entsprechenden Bromid gelingt, wegen der leichten Zersetzlichkeit des Rhodanids, ebenfalls nur in der Kälte.

Schmelzpunkt: $146^{\circ}$.

Wasser löst das Salz schwer mit hellblauer, Alkohol mit grünblauer Farbe. Die Reaktionen sind fast die gleichen, wie bei den beiden vorhergegangenen Kupferrhodaniden; nur gibt Ferrocyankalium einen braunroten, Ferricyankalium einen dunkelgrünen Niederschlag, die beide in der Hitze unlöslich sind.

Berechnet: $26.51 \% \mathrm{Cu} \quad 26.60 \% \mathrm{~S} \quad 25.02 \%$ en Gefunden: $26.72, \mathrm{Cu} \quad 26.51, \mathrm{~S} \quad 25.26$, en

b) Haloide, Acetate, Nitrate, Sulfate.

Monoäthylendiamin-Kupferchlorid. $\mathrm{CuCl}_{2}, 1$ en $+1 \mathrm{H}_{2} \mathrm{O}$.

Von den Additionsverbindungen des Kupferchlorid mit Äthylendiamin haben Wraner und SPruck ${ }^{1}$ bisher nur die Tetramminver-

${ }^{1}$ Z. anorg. Chem. 21 (1899), 234. 
bindung $\left(\mathrm{Cu}_{\left(\mathrm{H}_{2} \mathrm{O}_{2}\right.}^{\mathrm{en}}\right) \mathrm{Cl}_{2}$ dargestellt, die in leicht löslichen, rotblauen Blättern kristallisierte. Die Herstellung des Triäthylendiamin-Kupferchlorid ist nicht gelungen, denn es kristallisierten immer nur die Blätter der obigen Verbindung, die durch Analysen ${ }^{1}$ identifiziert wurden. Dagegen war die Darstellung des Diaminsalzes leicht; es kristallisierte aus molekularen Mengen Kupferchlorid und Äthylendiamin nach Konzentration der Lösung in feinen, hellblauen Nadeln. Schmelzpunkt: $158^{\circ}$.

Es ist in Wasser ziemlich leicht löslich, wird von Ammoniak, Kalilauge, Natriumphosphat und Natriumkarbonat nicht beeinflust, wäbrend Ferrocyankalium einen rotbraunen, Ferricyankalium einen dunkelgrünen und Jod in Jodkaliumlösung einen in der Hitze löslichen, grünen Niederschlag zeigt. Quecksilberchlorid gibt eine weilse Fällung. Durch Übergiefsen der Substanz mit konzentrierter Salzsäure entsteht eine gelbe Lösung, aus der beim Abkühlen das Doppelchlorid $\left(\mathrm{CuCl}_{4} \mathrm{CH}_{2} \mathrm{NH}_{3}\right)$ in goldgelben, prächtigen Blättel’n kristallisiert, mit dem Schmelzpunkt $272^{\circ}$.

$\begin{array}{llll}\text { Berecbnet: } 29.92 \% \mathrm{Cu} & 28.26 \% & \text { en } & 33.35 \% \mathrm{Cl} \\ \text { Gefunden: } 29.63 \% \mathrm{Cu} & 28.69 \% & \text { en } & 33.45 \% \mathrm{Cl} .\end{array}$

Triäthylendiamin-Kupferbromid. $\left(\mathrm{CuBr}_{2}\right) \cdot 3$ en $+5 \mathrm{H}_{2} \mathrm{O}$.

Setzt man zu einer konzentrierten Kupferbromidlösung 3 Mol. Äthylendiamin, so wird die Lösung tiefblau: aus ihr kristallisiert das Salz nach starker Konzentration in dunkelblauen, glänzenden Blättchen, die über Schwefelsüure getrocknet wurden.

Schmelzpunkt: $122^{\circ}$.

Das Salz zeichnet sich durch Beständigkeit aus, während das analoge Chlorid nicht darstellbar war. Es ist in Wasser leicht löslich und zeigt die gleichen Reaktionen wie das entsprechende Rhodanid dieser Reihe.

Berechnet: $12.88 \% \mathrm{Cu}$ $36.51 \%$ en $18.23 \% \mathrm{H}_{2} \mathrm{O}$ Gefunden: $12.69, \mathrm{Cu} 36.83$, en $17.91, \mathrm{H}_{2} \mathrm{O}$.

${ }^{1}$ Berechnet: $22.05 \% \mathrm{Cu}$ Gefunden: $21.77 \% \mathrm{Cu}$ $\left.\begin{array}{l}41.41 \% \text { en } \\ 41.18 \% \text { en }\end{array}\right\} \quad \mathrm{CuCl}_{2} \cdot 2 \mathrm{en}+2 \mathrm{aq}$. 
Diäthylendiamin-Kupferbromid.

$\left(\mathrm{CuBr}_{2}, 2 \mathrm{en}\right)+2 \mathrm{H}_{2} \mathrm{O}$.

Nach Zusatz der molekularen Menge Äthylendiamin zu käuflichem Kupferbromid entstand eine tiefblaue Lösung. Diese wurde bis zur Kristallhaut eingedampft; nach längerem Stehen kristallisierten aus ihr dunkelblane, prismatische Kristalle.

Schmelzpunkt: $126^{\circ}$.

Diese Verbindung ist in Wasser leicht und in Alkohol schwer löslich. Die übrigen Reaktionen sind die gleichen wie bei dem entsprechenden Diäthylendiamin-Kupferrhodanid.

$\begin{array}{llllll}\text { Berechnet: } 16.75 \% & \mathrm{Cu} & 42.14 \% \mathrm{Br} & 31.64 \% & \text { en } \\ \text { Gefunden: } 16.58, & \mathrm{Cu} & 42.32 \% \mathrm{Br} & 31.77 \% & \text { en. }\end{array}$

Monoäthylendiamin. Kupferbromid.

$$
\left(\mathrm{CuBr}_{2} .1 \mathrm{en}\right)+1 \mathrm{H}_{2} \mathrm{O} \text {. }
$$

In analoger Weise wie die vorher behandeiten Verbindungen geschah die Darstellung durch Zusatz von molekularen Mengen Äthylendiamin zu Kupferbromidlösung. Das Salz kristallisierte aus der stark konzentrierten Lösung in glänzenden, grünen, kleinen Platten.

Schmelzpunkt: $217^{\circ}$.

In heifsem Wasser ist es leicht, in Alkohol schwerer mit tiefblauer Farbe Iöslich. Abweichend von den Reaktionen der Tetramminund Hexamminsalze fällt aus der Lösung Kalilauge in der Hitze einen dunkelbraunen Niederschlag. Natriumkarbonat liefert einen in der Hitze unlöslichen, hellblauen Niederschlag. $\mathrm{HBr}$ gibt eine dunkelviolette Lösung, aus der beim Abkühlen das Doppelbromid $\left(\mathrm{CuBr}_{4} \underset{\mathrm{CH}_{2} \mathrm{NH}_{3}}{\mathrm{CH}_{2} \mathrm{NH}_{3}}\right)$ kristallisiert.

$\begin{array}{llll}\text { Berecbnet: } 21.09 \% \mathrm{Cu} & 19.92 \% \text { en } & 53.02 \% \mathrm{Br} \\ \text { Gefunden: } 21.33 \% \mathrm{Cu} & 19.62 \% \text { en } & 53.22 \% \mathrm{Br} \text {. }\end{array}$

Diäthylendiamin-Kupfernitrat.

$$
\left(\mathrm{Cu}\left[\mathrm{NO}_{3}\right]_{2} \cdot 2 \mathrm{en}\right)+2 \mathrm{H}_{2} \mathrm{O} \text {. }
$$

Von den Additionsverbindungen des Kupfernitrats mit Äthylen. diamin war bisher nur das Hexamminsalz bekannt, das nach WERNER und Spruck die Zusammensetzung $\left(\mathrm{Cu}\left(\mathrm{NO}_{3}\right)_{2} \cdot 3 \mathrm{en}\right)+2 \mathrm{aq}$ hat und in glänzenden, blauvioletten Schuppen kristallisiert. Leider gelang es 
nicht, die Monoverbindung darzustellen, da sich bei der Konzentration molekularer Mengen, merkwürdigerweise unter verschiedenen Bedingungen, immer grüne, unlösliche basische Kupfersalze ausschieden. Durch Zusatz von 2 Mol. Äthylendiamin zu stark konzentrierter Kupfernitratlösung aber kristallisiert das sehr beständige Tetremminsalz in sechsseitigen, glitzernden, dunkelblauen Blättchen aus, die in Wasser leicht, mit blauvioletter Farbe löslich waren.

Schmelzpunkt: $213^{\circ}$.

Berechnet: $18.49 \% \mathrm{Cu} \quad 34.88 \%$ en $\quad 10.46 \% \mathrm{H}_{2} \mathrm{O}$ Gefunden: $18.84, \mathrm{Cu} 34.99$,, en $10.11, \mathrm{H}_{2} \mathrm{O}$.

$$
\begin{aligned}
& \text { Triäthylendiamin-Kupferacetat. } \\
& \qquad \mathrm{Cu}\left(\mathrm{CH}_{3} \mathrm{COO}\right)_{2} \cdot 3 \mathrm{en}+2 \mathrm{H}_{2} \mathrm{O} .
\end{aligned}
$$

Das Hexamminsalz kristallisiert aus der dunkelblauen Lösung, die durch Zufügen von 3 Mol. Äthylendiamin zu einer Lösung von Kupferacetat entstanden ist, in dunkelblauen, vierseitigen Blättern aus, die sehr hygroskopisch sind, zwischen Filtrierpapier getrocknet und schnell analysiert wurden. Sie sind in Wasser leicht löslich. Schmelzpunkt: ca. $67^{\circ}$.

Berechnet: $15.98 \% \mathrm{Cu} \quad 45.30 \%$ en $30.16 \%$ Essigs. $9.049 \% \mathrm{H}_{2} \mathrm{O}$ Gefunden: $15.34, \mathrm{Cu} 44.79$, en 29.64 "Essigs. 8.623 " $\mathrm{H}_{2} \mathrm{O}$

$$
\begin{gathered}
\text { Diäthylendiamin-Kupferacetat. } \\
\mathrm{Cu}_{(}\left(\mathrm{CH}_{3} \mathrm{COO}\right)_{2} \cdot 2 \mathrm{en}+1 \mathrm{H}_{2} \mathrm{O} .
\end{gathered}
$$

Bei starker Konzentration der blauen Lösung aus 1 Mol. Kupferacetat und 2 Mol. Äthylendiamin kristallisiert diese Verbindung in hellblauen, anscheinend rhombischen Platten, die in Wasser ohne Zersetzung leicht löslich sind.

Schmelzpunkt: $115^{\circ}$.

Berechnet: $19.89 \% \mathrm{Cu} 37.58 \%$ en $37.52 \%$ Essigs. $5.629 \% \mathrm{H}_{2} \mathrm{O}$ Gefunden: 19.62, , $\mathrm{Cu} 37.44$, en 37.98, , Essigs. $5.12, \mathrm{H}_{2} \mathrm{O}$.

Monoäthylendiamin-Kupferacetat.

$$
\mathrm{Cu} .\left(\mathrm{CH}_{3} \mathrm{COO}\right)_{2}, 1 \text { en. }
$$

Setzt man zu einer konzentrierten Kupferacetatlösung $1 \mathrm{Mol}$. Äthylendiamin und engt die tiefblaue Lösung ein, so kristallisieren aus ihr dunkelgrüne, grofse, glänzende Kristalle von prismatischer Form, die in Wasser schwerer löslich sind als die vorigen. 
Schmelzpunkt: ca. $273^{\circ}$.

$\begin{array}{llll}\text { Berechnet: } 26.31 \% \mathrm{Cu} & 24.86 \% \text { en } & 48.82 \% & \text { Essigs. } \\ \text { Gefunden: } 26.07 \% \mathrm{Cu} & 25.31, \text { en } & 49.31 \% \text { Essigs. }\end{array}$

Monoäthylendiamin-Kupfersulfat.

$\left(\mathrm{CuSO}_{4} \cdot 1 \mathrm{en}\right)+3 \mathrm{H}_{2} \mathrm{O}$.

Auch diese Diamminverbindung fehlt in der Reihe der Sulfate, von denen WERnER und SPRuck das Tetrammin- und Hexamminsaiz dargestellt haben. Die Darstellungsweise ist der dieser Salze analog. Man setzt zu einer Kupfersulfatlösung 1 Mol. Äthylendiamin, konzentriert die Lösung und erhält die Diaminverbindung nach einigen Tagen als hellblaues, kristallinisches Pulver, welches aus mikroskopisch-feinen Nadeln bestand.

Die Verbindung zersetzt sich bei $260^{\circ}$ und ist in Wasser leicht löslich. Durch Zusatz von Jodkalium scheidet sich hauptsächlich Kupferjodür neben Jod aus, was wiederum für die geringe Beständigkeit der Kupfermonoäthylendiaminverbindungen spricht. Kaliumrhodanid gibt in einer kaltgesättigten Lösung sofort das entsprechende schwer lösliche Rhodanid, während sich in der Wärme unlösliches Rhodanür abscheidet. Das entsprechende Bromid ist grün und beständiger als das Rhodanid. KCN und Bromwasser entfärben die Lösung des obengenannten Salzes und weder $\mathrm{KOH}$, Ammoniak, noch Natriumkarbonat geben einen Niederschlag. Ferrocyankalium fällt einen braunen, Ferricyankalium einen gelbbraunen Niederschlag, die beide in der Hitze unlöslich sind.

\begin{tabular}{|c|c|}
\hline $\begin{aligned} & \text { t: } 23.23 \% \\
& \text { : } 23.59 \% \\
& \mathrm{Cu}\end{aligned}$ & $\begin{array}{ll}35.09 \% & \mathrm{SO}_{4} \\
35.44 & \mathrm{SO}^{2}\end{array}$ \\
\hline
\end{tabular}

Es folgt hier eine Tabelle der bekannten und neu dargestellten Kupferverbindungen mit einigen ihrer Eigenschaften.

(S. Tabelle, S. 18.)

Die Formeln sind im Sinne der WerNerschen Anschauungen formuliert, jedoch teilweise experimentell nicht bewiesen. Verbindungen, welche $2 \mathrm{Mol}$. Äthylendiamin neben Wasser enthalten, können von Verbindungen mit der Koordinationszahl 4 oder 6 abgeleitet werden. Der Begriff der Koordination läfst hier an Eindeutigkeit etwas zu wünschen übrig. 

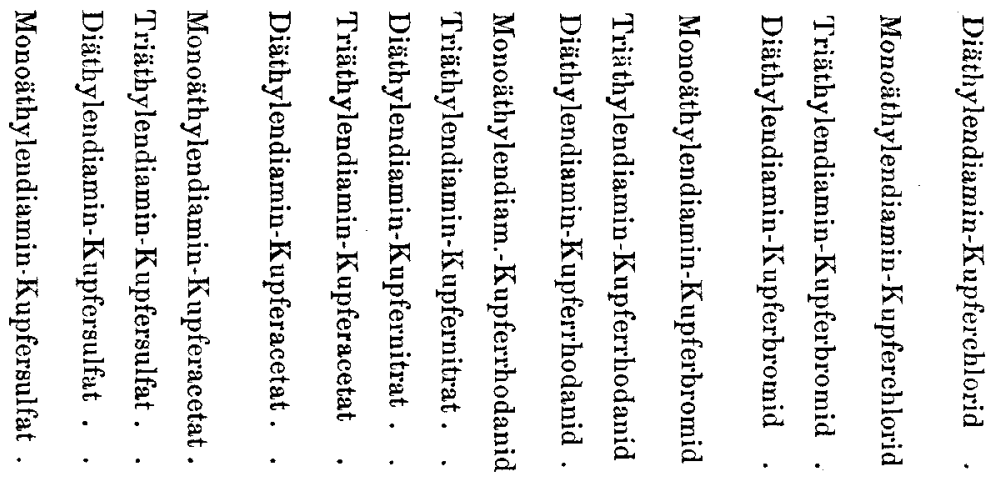

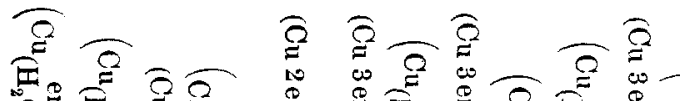

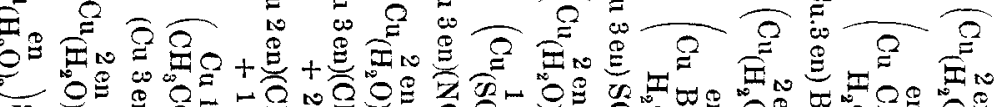
\%

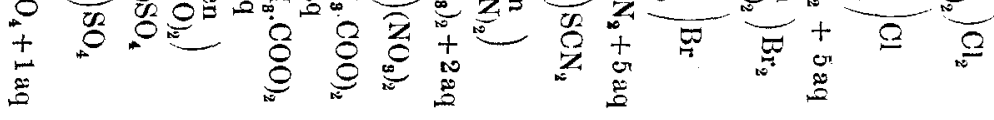

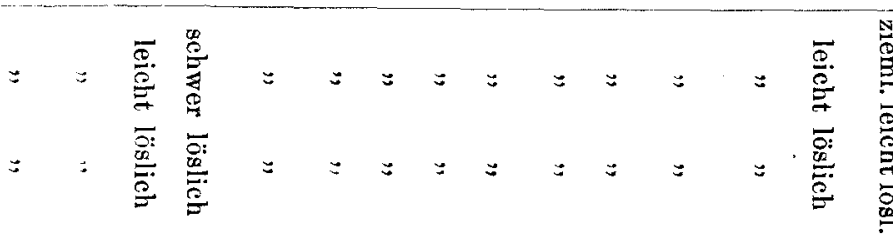

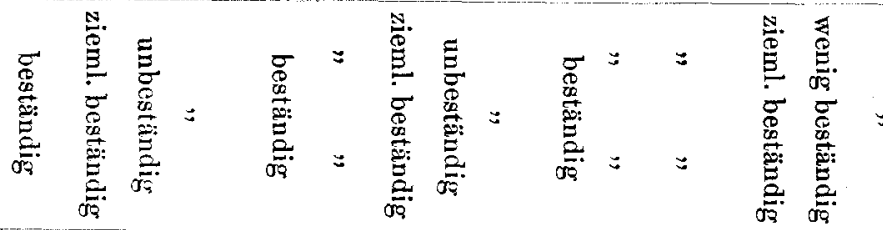

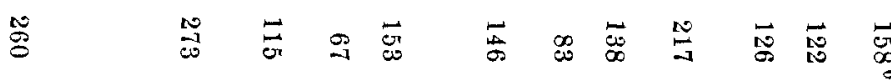

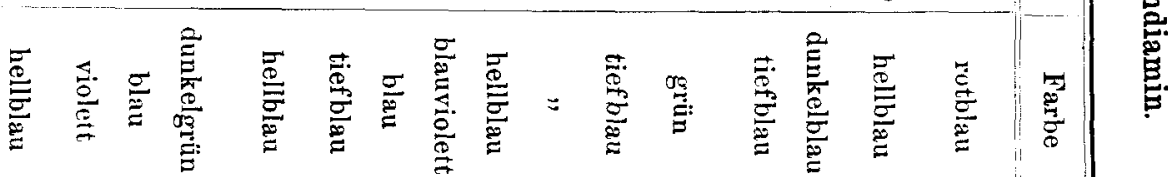

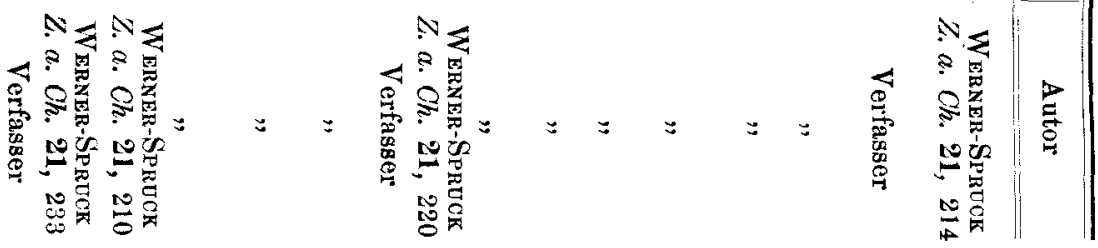
$\stackrel{\stackrel{0}{0}}{\stackrel{0}{\sigma}}$ ๑ั 总 品 罵 是 总 


\section{Anhang.}

\section{Einwirkung von $\alpha$ und $\beta$ Naphthylamin auf Metallrhodanid.}

Versuche zur Darstellung von Additionsverbindungen der beiden hochmolekularen Basen $\alpha$ und $\beta \mathrm{C}_{10} \mathrm{H}_{7} \mathrm{NH}_{2}$ an Metallrhodanide hatten nur geringen Erfolg. Der Grund ist teilweise in der zu verschiedenen Löslichkeit der Metallrhodanide und der Basen in Alkohol (Wasser ist nicht anwendbar) zu suchen.

Das schwer lösliche Naphthylamin, hauptsächlich indes die $\beta$-Verbindung, kristallisiert immer zuerst aus der alkoholischen Lösung und konnte von dem eventuell entstandenen Additionsprodukt schwer getrennt werden, da auch dieses in heifsem Alkohol löslich war. In einigen Fällen, so bei dem Versuch, die Additionsverbindungen der Quecksilber- und Kadmiumrhodanide mit $\alpha$ - und $\beta$-Naphthylamin darzustellen, wurde überhaupt keine Base an das Metallsalz angelagert, denn die ausgeführten Analysen bewiesen, dals nur reines Metallrhodanid neben der Base kristallisierte. Erfolg hatte nur die Darstellung der Additionsverbindungen von $\alpha$ - und $\beta$-Naphthylamin mit Nickelrhodanid.

Durch Zusatz von 2 Mol. $\alpha-\mathrm{Naphthylamin} \mathrm{in} \mathrm{alkoholischer}$ Lösung zur alkoholischen Lösung von Nickelrhodanid entstand sofort ein moosgrüner, kristallinischer Niederschlag, der abgesaugt und getrocknet wurde.

Das Salz war in Wasser, Alkohol und verdünnten Säuren in der Kälte unlöslich, in konzentrierten Säuren nur in der Hitze löslich. Es zersetzte sich bei $200^{\circ}$.

Nach 2 Nickelbestimmungen lag

$$
\left[\mathrm{Ni}\left(\alpha-\mathrm{C}_{10} \mathrm{H}_{7} \mathrm{NH}_{2}\right)\right] \mathrm{SCN}_{2} \text { vor. }
$$

Berechnet: $12.74 \%$ Ni. Gefunden: $13.12 \% \mathrm{Ni}$.

In analoger Weise wurde auch die Additionsverbindung des Nickelrhodanids mit $2 \mathrm{Mol} . \beta$-Naphthylamin dargestellt; sie fiel ebenfalls sofort als gelbgrüner, kristallinischer Niederschlag aus, dessen Löslichkeit mit der der $\alpha$-Verbindung übereinstimmte. Schmelzpunkt $261^{\circ}$ (unscharf).

Nach 2 Nickelbestimmungen scheint der Körper auf die Formel $\left[\mathrm{Ni}\left(\beta-\mathrm{C}_{10} \mathrm{H}_{7}, \mathrm{NH}_{2}\right)_{2}\right] \mathrm{SCN}_{2}$ zu stimmen.

Berechnet: $12.74 \% \mathrm{Ni}$.

Gefunden: $13.28 \%$ Ni. 
Weitgehende Schlüsse möchten wir aus diesen letzten Versuchen nicht ziehen.

Die Beziehungen, welche zwischen dem Bestehen fester Molekularverbindungen und den Eigenschaften des Zentralatoms, des Säureatoms und der organischen Basen vorhanden sind, sollen demnächst bei den Salzen der Ameisen und Essigsäure eingehend studiert werden. Die geehrten Fachgenossen bitten wir, uns dieses Gebiet für die nächste Zeit zur ungestörten Bearbeitung überlassen $\mathrm{zu}$ wollen.

Berlin N, Wissenschaftlich-chemisches Institut.

Bei der Redaktion eingegangen am 12. Mai 1906. 